Erschienen in: Blühdorn, Hardarik/Breindl, Eva/Waßner, Ulrich W. (Hrsg.):

Text - Verstehen. Grammatik und darüber hinaus. - Berlin, New York: de

Gruyter, 2006. S. 299-314. (Institut für Deutsche Sprache. Jahrbuch 2005), https://doi.org/10.1515/9783110199963.2.299

\author{
Selma M. Meireles
}

\title{
Leseverstehen aus der Perspektive des Nicht-Muttersprachlers ${ }^{1}$
}

\begin{abstract}
Dem Leseverstehen in der Mutter- und in der Fremdsprache liegt ein gemeinsamer Leseprozess zugrunde, der aber dennoch im Detail ganz unterschiedlich verlaufen kann. Es bestehen diesbezüglich erhebliche Unterschiede im Bereich des Wissens und des Grades der Automatisierung. Ein Mangel an sprachlichem, stilistischem und kulturellem Vorwissen erschwert für Fremdsprachenleser das Textverstehen; Leseheuristiken der Muttersprache können negative Interferenzen auslösen, und oft müssen syntaktische und morphologische Signale neu gewichtet werden. Das größte Problem für einen fremdsprachlichen Leser ist aber wohl, sich einen angemessenen, differenzierten und reichen Wortschatz anzueignen, der ein schnelles und effizientes Verständnis der Textbotschaft ermöglicht. Schriftliche Texte sind oft die einzige Kontaktmöglichkeit zur Zielsprache und -kultur außerhalb des Landes, in dem die Zielsprache gesprochen wird, jedoch sind für Nicht-Muttersprachler die oberflächlichen Lesestile oft nicht ausreichend. Solche Erkenntnisse können und sollen in die Lesedidaktik und in das Lesetraining in der Fremdsprache integriert werden.
\end{abstract}

\section{0. $\quad$ Einleitung}

Wenn jemand in seiner Muttersprache gut lesen kann, sollte dann nicht dasselbe auch für Fremdsprachen gelten, zumindest für solche, die man gut beherrscht? Hier wird exemplarisch gezeigt, dass dem Leseverstehen in der Muttersprache und in der Fremdsprache ein gemeinsamer Leseprozess zugrunde liegt, der aber dennoch im Detail ganz unterschiedlich verlaufen kann.

\section{1. „Lesen ist Lesen“- Lesen als Prozess}

In einem Text werden Inhalt und Kohärenz nicht durch bloße Dekodierung der sprachlichen Zeichen erzeugt. Sprachliche Texte, seien sie gesprochen oder geschrieben, erhalten ihre Bedeutung nur durch eine aktive Bearbeitung seitens des Rezipienten, in dessen Kopf komplexe mentale Prozesse ablaufen müssen, damit er aus den Zeichen einen Sinn entnehmen kann. Genau wie in

Mein Aufenthalt in Mannheim wurde vom Deutschen Akademischen Austauschdienst (Kennziffer A/05/15973) und von der Forschungsförderungs-Stiftung FAPESP des Bundeslandes São Paulo (Brasilien) gefördert (Vorgangs-Nr. 04/12767-5). Für die vielen kritischen Hinweise und wertvollen Anregungen danke ich Hardarik Blühdorn. 
einer mündlichen face-to-face-Kommunikation hängt der Erfolg des Leseverstehens ab von der Kooperation zwischen dem Produzenten, der seinen Text im Hinblick auf den Adressaten gestaltet, und dem Rezipienten, der sein allgemeines Wissen benutzt, um anhand der vom Produzenten gegebenen Hinweise die intendierten mentalen Repräsentationen aufzubauen. Bei dieser Rekonstruktion genügt aber keineswegs nur das sprachliche Wissen. Dazu kommen noch verschiedene Komponenten, wie inhaltliches Vorwissen, Kontexte, Situierungen und Erwartungen. Diese spielen beim Lesen eine unvorhersehbare, aber sehr wichtige Rolle, so dass es kein von vornherein feststehendes Verständnis eines Textes gibt und jeder Leser eine andere Wahrnehmung bzw. ein anderes Verständnis von einem Text hat. Dasselbe gilt auch für verschiedene sukzessive Lektüren eines Textes durch denselben Leser.

Die allgemeinen Bedingungen des Leseverstehens sind identisch für das Lesen in der Mutter- wie in einer Fremdsprache. Deshalb habe ich hier als erste These formuliert: „Lesen ist Lesen“. Die Vorgänge, die sich in beiden Lesesituationen abspielen, sind zunächst dieselben. Ein anschauliches Schema nach Börner/Vogel (1996, S. 5) illustriert die Leseprozesse, die mutter- und fremdsprachlichen Lesern gemeinsam sind:

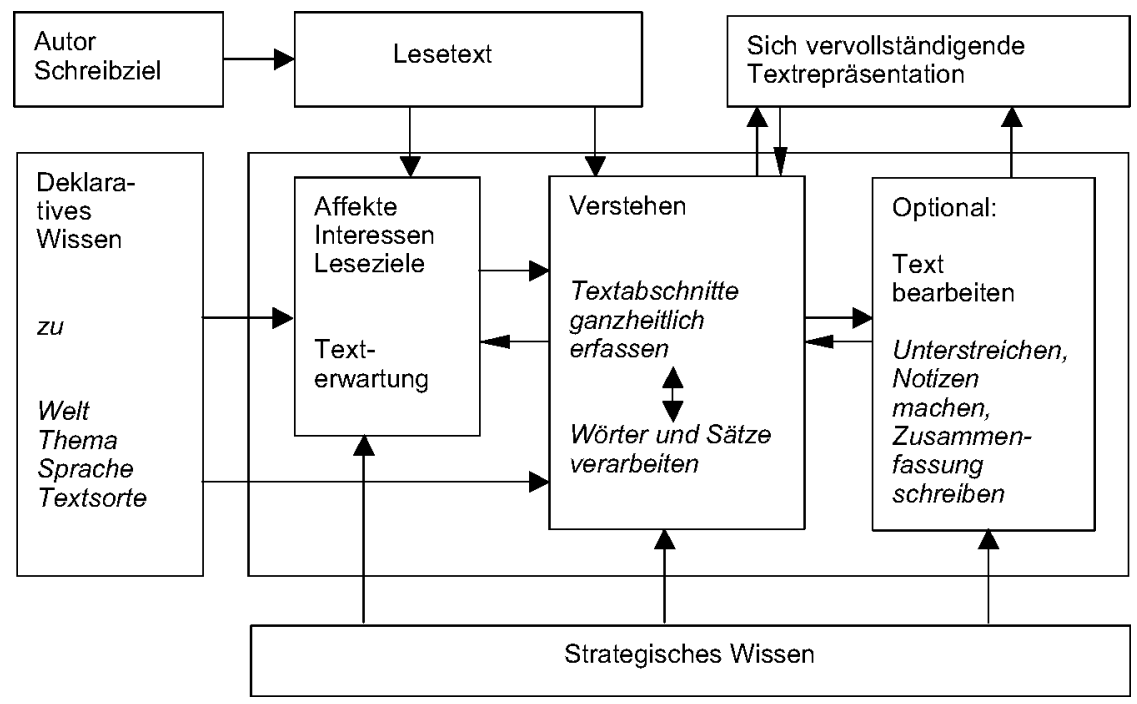

Abb. 1: Leseprozesse (nach Börner/Vogel 1996, S. 5)

Im Schema wird deutlich, dass es keine direkte Verbindung zwischen dem Text und seiner mentalen Repräsentation gibt. Diese Repräsentation kann nur durch die Interaktion der vielen anderen hier angegebenen Faktoren zustande kommen, und der Lesetext ist nur der Auslöser der involvierten Prozesse.

Auf jeder Ebene des Lesens sind zwei Hauptmomente festzustellen: das der Wahrnehmung und ein weiteres der Informationsverarbeitung. Groeben/ 
Christmann (1996) bezeichnen das Lesen als eine Wechselwirkung zwischen einem gegebenen Text und den Kognitionsstrukturen des Lesers. Die Textinformation wird je nach Zielsetzung, Interessen, individuellen Denkstilen usw. auf mehreren Ebenen ausgewertet und interagiert mit dem Wissen des Lesers.

\subsection{Sprach- und Weltwissen}

Obwohl sie in Wirklichkeit nicht voneinander zu trennen sind, kann man hier in heuristischer Absicht in Bezug auf das Leseverstehen zwei Wissenskomponenten unterscheiden: die Sprachwissens- und die Weltwissenskomponente. Die Wissenskomponenten entwickeln sich bei jedem Menschen unterschiedlich und sind außerdem kulturspezifisch geprägt (Wolff 1996, S. 546).

Das sprachliche Wissen umfasst das Wissen über Buchstabenkombinationen, morphologische und syntaktische Strukturen, semantische Eigenschaften von Morphemen und Wörtern sowie über die sprachliche Darstellung von logischen Operationen. Es ermöglicht die Wahrnehmung und Wiedererkennung der Hinweise zur Rekonstruktion der Mitteilung des Textes. Weist dieses Wissen Mängel auf, so hat das zur Folge, dass die sprachliche Dekodierung des Textes fehlerhaft, unangemessen oder gar unmöglich wird.

Das Weltwissen enthält zunächst einmal unser ganzes propositionales Wissen über die Welt. Dazu kommt sprachgebundenes Wissen wie Muster für die verschiedenen Textsorten, Routineformeln, die in bestimmten Situationen gebraucht werden usw. Ferner gehört zum Weltwissen auch Wissen, das oft nicht versprachlicht werden kann, wie Gefühle, Erfahrungen, Glauben usw., sowie Schemata und Strategien für das Verhalten in unterschiedlichen Situationen. Im Schema von Börner/Vogel werden Sprach- und Weltwissen unter der Rubrik „deklaratives Wissen“ zusammengefasst.

\subsection{Verarbeitungsprozesse}

Man kann zwei Typen von Verarbeitungsprozessen unterscheiden, die sich beim Textverstehen gleichzeitig abspielen: die aufsteigende Verarbeitung, auch datengeleitet oder bottom-up genannt, und die absteigende, wissensgeleitete oder top-down-Verarbeitung.

Bei der bottom-up-Verarbeitung geht der Leser von den einzelnen Elementen des Lesetextes, wie Wörtern und Sätzen, zur Erfassung des gesamten Textsinns über. Sprachkenntnisse sind immer die Grundlage für das Textverstehen. Es gibt aber auch eine sprachübergreifende Lesefähigkeit, die von der Muttersprache auf eine Fremdsprache transferiert werden kann.

Die top-down-Verarbeitung geht vom eigenen Wissen über die Welt und/ oder von der ganzheitlichen Erfassung des Textes aus. Das Vorwissen über die kommunikative Situation oder die formalen Konventionen einer bestimmten Textsorte z. B. führt zur Bildung von Erwartungen und Hypothesen über den Inhalt des Textes, die während der Lektüre durch die sprachlichen Daten bestätigt und/oder aktualisiert werden. 


\subsection{Lesestil}

Die Motivation und das Leseziel bestimmen die Art und Weise, wie gelesen wird. Das wird intuitiv klar, wenn man an sein eigenes Verhalten beim Lesen denkt. Ein Artikel über eine Theateraufführung wird z. B. anders gelesen je nachdem, welches Ziel man mit der Lektüre verfolgt. Wenn man nur wissen möchte, zu welcher Uhrzeit und an welchen Tagen das Stück aufgeführt wird, liest man ihn anders, als wenn man sich für die Handlung oder für Details der Inszenierung interessiert. Ob man selbst beruflich oder bloß zum Vergnügen mit dem Theater Kontakt hat, beeinflusst auch den Umfang und die Tiefe der Lektüre. Derselbe Text wird dann je nach Intention oder Haltung anders wahrgenommen und erfasst, und zwei Leser können dadurch sehr unterschiedliche Repräsentationen vom selben Text aufbauen.

Intentionen und Haltungen modifizieren die Problemlösung, führen zu Hypothesenbildung, die durch die Textverarbeitung bestätigt, ergänzt oder modifiziert wird, und bestimmen den Lesestil. In der fremdsprachendidaktischen Literatur hat sich eine Typologie von vier Lesestilen etabliert, die von den Zielen bestimmt werden, die der Leser beim Lesen verfolgt. Häussermann/Piepho stellen sie in ihrem Aufgabenbuch Deutsch als Fremdsprache (1996) vor.

Der erste Kontakt mit dem Text geschieht durch das Überfliegen, auch als globales oder orientierendes Lesen bekannt. Auffällige Merkmale des Textes wie Überschrift, fettgedruckte Ausdrücke, Schlüsselwörter werden auf schnelle Weise aufgenommen. Auf dieser Basis gewinnt der Leser eine erste Vorstellung vom Inhalt. Ein alltägliches Beispiel dafür wäre die schnelle, überfliegende Lektüre der ersten Seite einer Tageszeitung. Dabei wird entschieden, ob ein Text interessant ist. Wenn ja, wird unter den folgenden Lesestilen gewählt.

Beim Mustern oder kursorischen Lesen werden der Aufbau, der Zusammenhang und die Hauptaussagen erfasst. Das kursorische Lesen ermöglicht ein relativ schnelles, aber dennoch nicht ganz oberflächliches Textverständnis, so dass der Leser die für ihn neuen oder interessanten Thesen aufnehmen kann. Wenn wir bei unserem Zeitungsbeispiel bleiben, so wird dieser Lesestil bei der schnellen Lektüre der Berichte über aktuelle Tagesereignisse angewendet.

Ein anderer, alternativer Lesestil für die schnelle Entnahme von Information ist das Herauspicken (auch als selegierendes oder selektives Lesen bekannt). Hier hat der Leser schon vor dem Beginn der Lektüre ein bestimmtes Ziel. Es werden gezielt nur die Informationen herausgeholt, die für einen bestimmten Zweck wichtig sind. In der Zeitung hätten wir diesen Lesestil z. B. bei der Lektüre der Wettervorhersage, bei der normalerweise nur auf die Vorhersage für einen bestimmten Ort geachtet wird.

Wenn man sich dafür entscheidet, noch tiefer in den Text einzudringen, z. B. bei der Lektüre des Leitartikels, handelt es sich um das Verweilen oder das 
detaillierte Lesen. Hier werden der Text und seine Botschaft so sorgfältig wie möglich aufgenommen, sei es analytisch-kritisch (z. B. ein Gerichtsurteil), umsetzend (z. B. eine Gebrauchsanweisung) oder genießend (z. B. ein Gedicht) (vgl. Häussermann/Piepho 1996).

Es gibt hinsichtlich der mentalen Prozesse und der Faktoren, die dabei eine Rolle spielen, keinen Unterschied zwischen dem Lesen in der Mutter- oder in einer Fremdsprache. In beiden müssen Wörter, Sätze und Textabschnitte erfasst und verarbeitet werden, damit man zu einer mentalen Textrepräsentation gelangt. Es bestehen jedoch erhebliche Unterschiede im Bereich des Wissens und des Grades der Automatisierung der involvierten Prozesse. Für Nicht-Muttersprachler sind außerdem die oberflächlichen Lesestile oft nicht ausreichend bzw. führen zu keinem, nicht einmal einem oberflächlichen Textverständnis. Das bringt uns zu der zweiten These.

\section{2. „Fremdsprachler haben es schwerer" - Lesen in einer Fremdsprache}

Die intendierten mentalen Repräsentationen werden dadurch gebildet, dass die sprachlichen Informationen, die im Text enthalten sind, vom Leser anhand seines Repertoires an sprachlichem und außersprachlichem Wissen erfasst, verarbeitet und ergänzt werden. In einem Text wird nicht explizit alles gesagt, was verstanden werden kann oder soll.

\subsection{Sprachliches Vorwissen}

Beim Textverstehen muss der Leser „Schriftzeichen und ihre Kombinationen sowie satzgliedernde Elemente wie die Zeichensetzung erkennen, ihnen die angemessene Bedeutung zuordnen, die Regelhaftigkeit des Satzbaus erkennen und korrekt interpretieren " (Solmecke 1993, S. 13). Dazu kommen Vokabelkenntnisse, die den Leser befähigen, Bedeutungen möglichst automatisch abzurufen und Inferenzen zu ziehen. Vokabelkenntnisse sind im Endeffekt der wichtigste Faktor beim Lesen.

De facto kann man dem Wortschatz eine besonders prominente Rolle im Leseverstehen zuschreiben. Wortbedeutungen bestehen aus verschiedenen Komponenten, wie dem begrifflichen Gehalt, Situationsschemata und affektiven Färbungen, die gemeinsam im mentalen Lexikon gespeichert sind. Zum Beispiel wird bei dem Wort „Hund“ neben der lautlichen und graphemischen Form des Wortes eine Vorstellung davon gespeichert, was ein prototypischer Hund ist, z. B. ein vierbeiniges, fleischfressendes, bellendes Haustier. Hinzu kommt z.B. das Wissen, dass Begegnungen mit Hunden manchmal gefährlich sein können, und/oder Abneigungen gegen bzw. Vorlieben für bestimmte Hundeindividuen. Die Psycholinguistik unterscheidet hier zwischen enzyklopädischem und episodischem Wissen. Während das episodische Wissen grundsätzlich individuell ist, wird ein großer Teil des enzyklopädischen Wissens von einer ganzen Sprach- und Kulturgemeinschaft geteilt. 
Das größte Problem für einen fremdsprachlichen Leser ist dann wohl, sich einen angemessenen, differenzierten und reichen Wortschatz anzueignen, damit er die vom Verfasser intendierte Botschaft schnell und effizient verstehen kann. In einer unveröffentlichten Untersuchung der Universität São Paulo mit jugendlichen Deutschlernern zeigte sich, dass ca. $85 \%$ der Probanden eine Liste der Schlüsselwörter eines Textes als die effektivste Hilfe beim Textverständnis empfinden, weit vor anderen Hilfsmitteln wie Paralleltexten in der Mutter- oder Fremdsprache, Bildern, Graphiken und Aktivierung der Vorkenntnisse durch den Lehrer (Rogério 2003).

Auch syntaktische Informationen müssen automatisch aufgenommen werden, denn ein Satz ist mehr als die Summe der Wortbedeutungen. Bei der Dekodierung eines Satzes werden zugleich seine inhaltlichen wie auch seine syntaktischen Informationen aufgenommen, aber viele sprachliche Hinweise werden von fremdsprachlichen Lesern einfach nicht angemessen wahrgenommen.

Ein wichtiges Beispiel dafür bietet die Unterscheidung zwischen Subjekt und Objekt, besonders bei Topikalisierung, wie im folgenden Satz:

\section{(1) Die Chefsekretärin hat der Assistent nicht rechtzeitig erreicht.}

Sehr oft wird in solchen Fällen der Definitartikel im Nominativ - wie bei der Assistent-vom nicht-muttersprachlichen Leser einfach übersehen, so dass die Chefsekretärin als Subjekt interpretiert wird.

Weitere heikle Punkte sind die Unterscheidung zwischen direkten und indirekten Objekten, insbesondere wiederum bei Topikalisierung, wie in:

(2) Der Chefsekretärin hat der Direktor eine Assistentin zugeteilt.,

ferner auch der Unterschied zwischen präpositionalem Objekt und präpositionalem Attribut, wie in:

\section{(3) Er hat die Tür mit dem Eisengitter geöffnet.}

Solche Beispiele lassen sich nur durch genaue Erfassung und Berücksichtigung ihrer morphosyntaktischen Eigenschaften und der Bedeutung der beteiligten Wörter angemessen dekodieren. Fabricius-Hansen berichtet in einer Arbeit von 2002 über ein Leseexperiment mit norwegischen Deutschlernenden, bei dem sich herausstellte, dass gerade die morphosyntaktischen Eigenschaften bei der Lektüre deutscher Texte oft vernachlässigt werden. Fremdsprachenleser verfügen schon über ein Repertoire an morphosyntaktischen, textuellen und situativen Mustern aus der Muttersprache, die sie unbewusst bei der Lektüre von fremdsprachlichen Texten einsetzen. Bei Anfängern ist eine solche Übertragung meist die einzige Möglichkeit, sich einem fremdsprachlichen Text zu nähern. Bei Fortgeschrittenen werden die muttersprachlichen Wissensstrukturen mehr und mehr durch die der Fremdsprache ersetzt oder ergänzt. Aber es besteht immer ein großes Risiko der negativen Interferenz. Fabricius-Hansen berichtet z. B., dass dem finiten Verb nachgestellte 
Subjekte systematisch nicht als Agens erkannt werden, da Norwegisch eine überwiegende $\mathrm{V}(\mathrm{erb}) \mathrm{O}$ (bjekt)-Struktur habe. Norwegische Deutschlernende benutzen dieses auch im Deutschen mögliche Satzmuster als Verstehenshypothese bei der Lektüre und landen oft bei falschen Interpretationen. Für ein besseres Leseverstehen im Deutschen müssten norwegische Muttersprachler ihre gewohnten Leseheuristiken ablegen, die Gewichtung der syntaktischen Signale ändern und morphologischen Signalen den Vorrang geben. Außerdem müssten sie die Kommasetzung höher einstufen, das Arbeitsgedächtnis auf OV-Strukturen trainieren und der strukturellen Vielfalt des Deutschen mit angemessener rezeptiver Flexibilität begegnen (vgl. Fabricius-Hansen 2002, S. 14).

Dieser Befund stimmt mit meiner Erfahrung bei brasilianischen Deutschlernenden überein. Auch Portugiesisch ist eine VO-Sprache und es gibt kaum morphologische Rektionsindikatoren, so dass Deklinationsendungen im Deutschen gern übersehen und flexiblere Satzmuster selten erkannt werden.

\subsection{Stilistisches Vorwissen}

Beim Schreiben berücksichtigt der Verfasser mehr oder weniger bewusst, dass er sich so ausdrücken muss, dass der Leser dem Text die Mitteilung auf eine ökonomische Weise entnehmen kann. Nach Solmecke (1993, S. 25) antizipieren Produzenten die Verstehensbedingungen und -voraussetzungen des Rezipienten und verwenden adressatengerichtete konventionalisierte Formen. Dafür müssen sie jedoch möglichst genaue Vorstellungen vom Hintergrundwissen des Lesers haben. Fabricius-Hansen (2002, S. 6) erinnert daran, dass es schon schwierig genug ist, wenn man es mit einem kollektiven, anonymen Leserkreis zu tun hat; die Bedürfnisse oder das Hintergrundwissen von potenziellen nicht-muttersprachlichen Lesern werden dabei normalerweise nicht berücksichtigt.

Auch bei einem muttersprachlichen Publikum kann der Autor nicht ganz sicher sein, dass er sein Schreibziel erreicht. Jedoch werden die Chancen dafür durch gemeinsame Vorstellungen und Erwartungen bezüglich konventionalisierter Text-, Schreib- und Interaktionsformen erhöht. Solche Konventionen unterliegen in verschiedenen Kulturen großen Unterschieden. Dadurch kann das Verstehen erschwert werden oder scheitern, denn das Wissenssystem des Lesers bestimmt die Wahrnehmung und Interpretation der sprachlichen Daten. Hier liegt ein weiterer Stolperstein für den fremdsprachlichen Leser, denn ihm fehlt oft die Kenntnis der stilistischen Muster und Textkonventionen, die sich von denen seiner Muttersprache unterscheiden.

Außerdem können fremdsprachliche Leser je nach ihrer Erfahrung mit der Zielsprache manche stilistischen Nuancen nicht adäquat verfolgen, wie z. B. die Unterschiede zwischen Standardsprache und Dialekt, Umgangssprache und Schriftsprache. Für sie kann zum Beispiel der Witz einer Version von Rotkäppchen in Amtsdeutsch verlorengehen. Auf dieselbe Weise sind viele Asso- 
ziationen, Gefühle und Erwartungen, die für einen Muttersprachler z.B. durch die Sprechweise einer Romanfigur hervorgerufen werden können, einfach nicht vorhanden, was das Textverständnis nicht unmöglich macht, aber unter Umständen erheblich reduzieren kann.

\subsection{Kulturelles Vorwissen}

Sprachkenntnisse sind eine notwendige, aber nicht hinreichende Bedingung zum Textverstehen. So z. B. beim folgenden Text:

(4) Markus hat in der Waschanlage vergessen, die Fenster zu schließen. Er musste schnell wieder nach Hause.

Dieser Text kann schon von Muttersprachlern unterschiedlich interpretiert werden. Einige Beispiele:

1. Markus war mit seinem Auto in der Waschanlage. Er saß während des Waschens im Auto. Da er vergessen hatte, die Fenster zu schließen, ist er nass geworden und muss nach Hause gehen, um sich umzuziehen.

2. Markus war mit seinem Auto in der Waschanlage. Er hat vergessen, die Fenster zu schließen, weil er (aus irgendwelchen anderen Gründen) schnell wieder nach Hause gehen musste.

Diese zwei Interpretationen unterscheiden sich in der Richtung der Kausalverknüpfung: in der ersten ist das Vergessen die Ursache für die Notwendigkeit wieder nach Hause zu gehen. In der zweiten ist die Notwendigkeit schnell nach Hause zu gehen die Ursache für das Vergessen.

Eine dritte mögliche Interpretation wäre etwa:

3. Markus arbeitet in einer Waschanlage. Er hat vergessen, die Fenster der Waschanlage zu schließen, weil er (aus irgendwelchen Gründen) schnell wieder nach Hause gehen musste.

Man kann auch die zwei Sätze des Textes als voneinander unabhängig verstehen, wenn man annimmt, dass es sich um ein Fragment eines längeren Textes handelt, der z. B. den Titel Ein schrecklicher Tag tragen könnte:

(5) Heute war ein schrecklicher Tag. Markus hat in der Waschanlage vergessen, die Fenster zu schließen. Er musste schnell wieder nach Hause. Dann hat er den Termin beim Zahnarzt verpasst. Im Büro ist sein Computer kaputt gegangen, und er hat einen ganzen Bericht verloren. Am Abend hatte er Krach mit seiner Freundin.

Diese und andere mögliche Interpretationen können sich sowohl bei einem muttersprachlichen als auch bei einem fremdsprachlichen Leser einstellen. Beide benötigen weitere Informationen aus dem Text oder aus dem kommunikativen Kontext, um die semantische Verknüpfung der beiden Sätze zu konstruieren. 
Asyndetische Satzverkettungen wie in unserem Beispiel kommen selten isoliert vor, sondern meist in Kontexte eingebettet, die die Disambiguierung erleichtern. Für einen fremdsprachlichen Leser stellt sich unter Umständen jedoch noch eine ganz andere Frage, deren Antwort für das Weiterlesen unentbehrlich ist: Was ist überhaupt eine Waschanlage?

Aus dem Wort allein kann man nicht entnehmen, was eine Waschanlage ist oder wie sie funktioniert. Man kann an der Wortbildung erkennen, dass es um einen Ort oder ein Gerät geht, an dem oder mit dem etwas gewaschen wird. Ohne weiteres kulturelles Wissen bleibt das Wort aber trotzdem undurchsichtig. Es lässt sich rein linguistisch weder von einer Waschmaschine noch von einer Spülmaschine unterscheiden.

Auch wenn erklärt wird, dass in einer Waschanlage Autos gewaschen werden, bedeutet das noch nicht, dass unser Beispieltext von Mitgliedern anderer Kulturgemeinschaften ohne weiteres adäquat verstanden werden kann. In der Stadt São Paulo sind Autowaschanlagen bekannt, aber sie unterscheiden sich von den deutschen in vielen Aspekten. Im Hinterland Brasiliens dagegen kann man keineswegs voraussetzen, dass die Menschen sich unter einer Autowaschanlage etwas vorstellen können.

Auch wenn man alle Wörter versteht und die syntaktischen Funktionen richtig erkennt, kann man den kurzen Text nicht als zusammenhängende Mitteilung erfassen, wenn man nicht weiß, dass in einer Waschanlage Autos gewaschen werden, und wie so eine Anlage aussieht und betätigt wird. Ohne diese Kenntnisse kann man die Erwähnung von Fenstern in diesem Kontext nicht verstehen und auch keine der vorher angedeuteten Interpretationen entwickeln.

Das kulturelle Wissen, d.h. die Erfahrungen, die man durch direkte oder von Medien geleitete Erlebnisse in Bezug auf die Zielkultur und ihre Sitten, Werte und Lebensweise gesammelt hat, beeinflusst die Erfassung, Interpretation und Zuordnung der im Text enthaltenen Begriffe. Vor kurzem haben Deutschstudenten bei mir im Unterricht einen Text über die Deutsche Bahn gelesen und Aufgaben zum Textverständnis bearbeitet. Es zeigte sich, dass sie beim Leseverstehen dem Vergleich zwischen dem Auto und der Bahn als Verkehrsmittel gut folgen konnten. Als sie jedoch aufgefordert wurden, selbst einen Verkehrsmittelvergleich zwischen Bahn und Bus anzustellen, interpretierten einige „Bahn“ als „U-Bahn“ und nicht als „Eisenbahn“. Sie bezogen ihren Vergleich also auf den Stadtverkehr und nicht auf den Fernverkehr. Dahinter steht offensichtlich die Verkehrsrealität Brasiliens, wo die Eisenbahn für den Personenfernverkehr kaum in Gebrauch ist.

Die Fähigkeit, mehr zu verstehen, als im Text steht, beruht auf dem Wissen über Situationen, Routinehandlungen, Rollenverhalten von Menschen, Kausalitäten, aber auch Textkonventionen, was zu Inferenzen und Antizipationen führt (Solmecke 1993, S. 18). Konventionalisierte Schemata auf allen Ebenen des Textes öffnen in den vorhandenen abstrakten Vorwissensstrukturen des Lesers ständig Leerstellen, bevorzugen die Aufstellung von bestimmten Er- 
wartungen und blenden schon sämtliche anderen Möglichkeiten aus. Außerdem ermöglichen sie, wichtige Informationen von weniger Wichtigem in einem Text zu unterscheiden. Dadurch wird eine geringere Menge von Informationen aufgenommen, behalten und im Gedächtnis gespeichert, was zu Verarbeitungsökonomie und erhöhter Lesegeschwindigkeit führt.

\subsection{Leseverstehen in der Fremdsprache: Zusammenfassung}

Bei der daten- und wissensgeleiteten Verarbeitung geht es um das Einsetzen von Informationen aus dem Text in die verschiedenen schematischen Sprachund Weltwissensstrukturen des Lesers. Hierfür muss man konventionalisierte Muster erkennen und Verbindungen mit den vorhandenen Kenntnissen auf eine schnelle, automatisierte Weise herstellen, damit das Arbeitsgedächtnis nicht überlastet wird. Unerfahrene muttersprachliche Leser haben schon genug Schwierigkeiten dabei, wie bei den Pisa-Studien klar geworden ist, aber Fremdsprachenleser haben es sozusagen noch schwerer.

Erstens benötigen sie je nach Sprachniveau eine relativ längere Zeit für die sprachliche Rekodierung, d.h. für das Erkennen von Buchstaben-, Wörterund Phrasenkombinationen und ihre semantische Zuordnung zu größeren Einheiten. Bei Anfängern ist diese Phase besonders kritisch, denn sie verfügen noch über einen zu geringen und wenig differenzierten Wortschatz und haben die fremdsprachlichen Muster noch nicht genügend automatisiert. Deshalb können sie nur selten Erwartungen bilden und müssen tatsächlich fast alle Informationen aus dem Text selbst entnehmen. Dadurch müssen sie den größten Teil ihrer Verarbeitungskapazität grundlegenden bottom-up-Prozessen widmen statt dem inhaltlichen Erfassen, was das Lesen mühsam und fragmentär macht, denn die Zahl der notwendigen Rekursionen ist viel höher als bei Fortgeschrittenen oder muttersprachlichen Lesern.

Dazu zeigt sich bei Fremdsprachlern im Allgemeinen auch ein Mangel an Hintergrundwissen im Bereich von Wortschatz, stilistischen Normen und Textkonventionen, sowie den kulturellen Grundlagen, die die Voraussetzung für das Einsetzen von top-down-Verfahren sind. Fabricius-Hansen (2002, S. 9f.) fasst die Situation sehr klar zusammen:

\footnotetext{
„Hinreichende Sprachkenntnisse und hinreichendes Hintergrundwissen garantieren insgesamt noch nicht, dass das Lesen in der Fremdsprache in optimaler Weise abläuft. Das setzt voraus, dass bottom-up- und top-down-Verfahren angemessen interagieren und dass die verkürzenden Leseheuristiken, die mit dem schemageleiteten Lesen einhergehen, der Fremdsprache angepasst sind. [...] Sie müssen die in fremdsprachlichen Texten wiederkehrenden Muster internalisiert haben und grammatische, cues" wie Flexionsendungen und Wortstellung korrekt gewichten."
}

Zusammenfassend können wir sagen, dass Mangel an sprachlichem, stilistischem und kulturellem Vorwissen für Fremdsprachenleser das Textverstehen stark erschwert. Die angemessenen Erwartungen und top-down/bottom-upVerarbeitungen werden nicht automatisch genug aktiviert, überprüft und ein- 
gesetzt, was die Lesegeschwindigkeit und die analytische Wahrnehmung des Inhalts erheblich beeinträchtigt.

Trotz der vielen Schwierigkeiten spielen jedoch schriftliche Texte für fremdsprachliche Leser wohl eine relativ größere Rolle als für die meisten Muttersprachler. Fremdsprachenlernende außerhalb des Landes, in dem die Zielsprache gesprochen wird, befinden sich in außerunterrichtlichen Situationen eher in der Rolle des Lesers, seltener oder gar nicht in der des Sprechers oder Hörers. Oft sind geschriebene Texte, auch aus dem Internet, die einzige Kontaktmöglichkeit zur Zielsprache und -kultur.

Außerdem hat die Entfernung des zielsprachigen Alltags großen Einfluss auf den geforderten Lesestil: Während der muttersprachliche Leser öfter mit Situationen konfrontiert wird, die das selegierende Lesen erfordern, wird der durchschnittliche Fremdsprachenleser öfter das detaillierte Lesen bevorzugen. Um ein konkretes Beispiel zu nennen: Nach Umfragen unter brasilianischen Deutschstudenten lernen Brasilianer die deutsche Sprache hauptsächlich aus zwei Gründen: Entweder brauchen sie die Sprache beruflich, etwa bei einem der vielen deutschen Unternehmen, die im Land eine Filiale haben, oder sie suchen einen engeren Kontakt zur deutschen Literatur, Musik oder Philosophie. In beiden Fällen werden sie mit Texten umgehen, die ein vertieftes Leseverstehen erfordern, seien es Fachtexte, Gebrauchsanweisungen oder literarische Werke.

\section{3. „Neu lesen lernen?“ - Lesetraining im Fremdsprachen- unterricht}

\subsection{Richtlinien für das Lesetraining in der Fremdsprache}

Wie können nun diese Erkenntnisse in die Lesedidaktik integriert werden? Jeder Fremdsprachenlehrende stellt sich beständig die Frage: Wie kann man die Lernenden dabei unterstützen, ihr eigenes Potential beim Lesen in der Fremdsprache zu erkennen und weiterzuentwickeln?

Auf der einen Seite kann die Leseheuristik der Muttersprache negative Interferenzen auf das Lesen in der Fremdsprache ausüben, wie in dem zitierten Beispiel von Fabricius-Hansen, wenn sich die angemessenen Strategien in beiden Sprachen unterscheiden und solche Unterschiede von den Lernenden nicht wahrgenommen und berücksichtig werden. Auf der anderen Seite ist es auch oft so, dass Lernende das Lesen in der Fremdsprache als ein völlig neues Verfahren betrachten und dabei die schon aus dem Lesen in der Muttersprache bekannten und erfolgreichen Strategien gerade nicht einsetzen und sich einer Art „atomistischem Lesen“ (Häussermann/Piepho 1996) hingeben, bei dem sie glauben, jedes Wort und jedes morphosyntaktische Merkmal bewusst und analytisch erfassen zu müssen, um einen fremdsprachlichen Text $\mathrm{zu}$ verstehen, was natürlich zu Systemüberlastung und Leseblockade führt.

Beim Lesen sowohl in der Mutter- als auch in der Fremdsprache werden Verfahren eingesetzt, die entweder unbewusst bei top-down- und bottom-up- 
Verarbeitungen aktiviert werden oder bewusst bei der Aufgaben- und Problemlösung eingesetzt werden. Hier kann man mit Ehlers zwischen kognitiven Strategien (wie z.B. zusammenfassen, inferieren, Hypothesen bilden und testen, Gebrauch von allgemeinem Wissen) und eigentlichen Lesetechniken unterscheiden (wie markieren, Stichwörter aufschreiben, Flussdiagramme erstellen usw., auch der Erzeugung von Paralleltexten zur Fixierung und/oder Veranschaulichung der Verarbeitungsprozesse) (vgl. Ehlers 2003, S. 288). Solche Strategien können und sollen auch im Lesetraining im Fremdsprachenunterricht eingeführt und geübt werden, um den Lernenden Werkzeuge anzubieten, die ihnen das autonome Lesen in der Zielsprache erleichtern.

Als Ausgangspunkt des Lesetrainings im Fremdsprachenunterricht soll es darum gehen, den Lernenden die aus der Muttersprache bekannten aber schon automatisierten, unbewusst eingesetzten Strategien wieder bewusst zu machen, sie den Erfordernissen der Fremdsprache anzupassen und in ihrer neuen Form wiederum zu automatisieren. Dabei muss man den Lernenden ständig Möglichkeiten anbieten, sich mit verschiedenen Texten und Textsorten zu befassen, ihren Wortschatz zu erweitern und zu differenzieren und textuelle und stilistische Muster kennenzulernen.

Zum Abschluss möchte ich als Beispiel einige Überlegungen präsentieren, die der Konzeption eines Lesekurses für Germanistikstudenten an der Universität São Paulo zugrundeliegen.

\subsection{Der Textproduktions- und -reproduktionskurs an der Universität São Paulo}

Die Universität São Paulo ist eine der wenigen Universitäten in Brasilien, an denen das Fach Deutsch studiert werden kann, und die einzige, die einen Magister- und Promotionsstudiengang in Germanistik anbietet. Von den zur Zeit etwa 350 Germanistiksstudenten des Graduierungsstudiums haben weniger als $10 \%$ ihr Studium mit Vorkenntnissen der deutschen Sprache begonnen. Es handelt sich eigentlich um eine Kombination von Deutsch als Fremdsprache mit Literaturgeschichte sowie Unterricht in Grammatik und Pragmatik der deutschen Sprache.

Da die kritische Auseinandersetzung mit deutschen literarischen und wissenschaftlichen Texten im Original zu den Zielen des Studiums gehört, müssen die Studenten schon bei relativ geringen Deutschkenntnissen in Kontakt mit solchen Texten kommen. Um sie dabei zu fördern, wurde eine PflichtLehrveranstaltung eingeführt, die „Textproduktion und -reproduktion“ genannt wird. Sie wird nach ca. 150 Stunden Deutschunterricht besucht und umfasst vier Semester-Wochenstunden. Es handelt sich um einen Lese- und Schreibkurs, in dem versucht wird, die hier skizzierten Richtlinien anhand der Bearbeitung von deutschen Originaltexten zu verfolgen: Bewusstmachung und Automatisierung der Lesestrategien und Erweiterung des Wortschatzes sowie der allgemeinen Sprachkompetenz. 
Die Arbeit wurde so konzipiert, dass jede Unterrichtseinheit sich mit einem anderen Text bzw. einer anderen Textgruppe beschäftigt und ein Hauptlernziel hat. Als Erstes geht es um die Bewusstmachung der Existenz verschiedener Lesestile und Textmuster. Ich führe zum Abschluss ein Beispiel aus der ersten Unterrichtseinheit vor.

Damit die Studenten nicht um jeden Preis versuchen, einen zu lesenden Text Wort für Wort zu dekodieren, werden sie zunächst mit einem Text in einer ihnen ganz unbekannten Sprache konfrontiert, z. B. einem polnischen Artikel über die Formel 1 aus dem Internet:

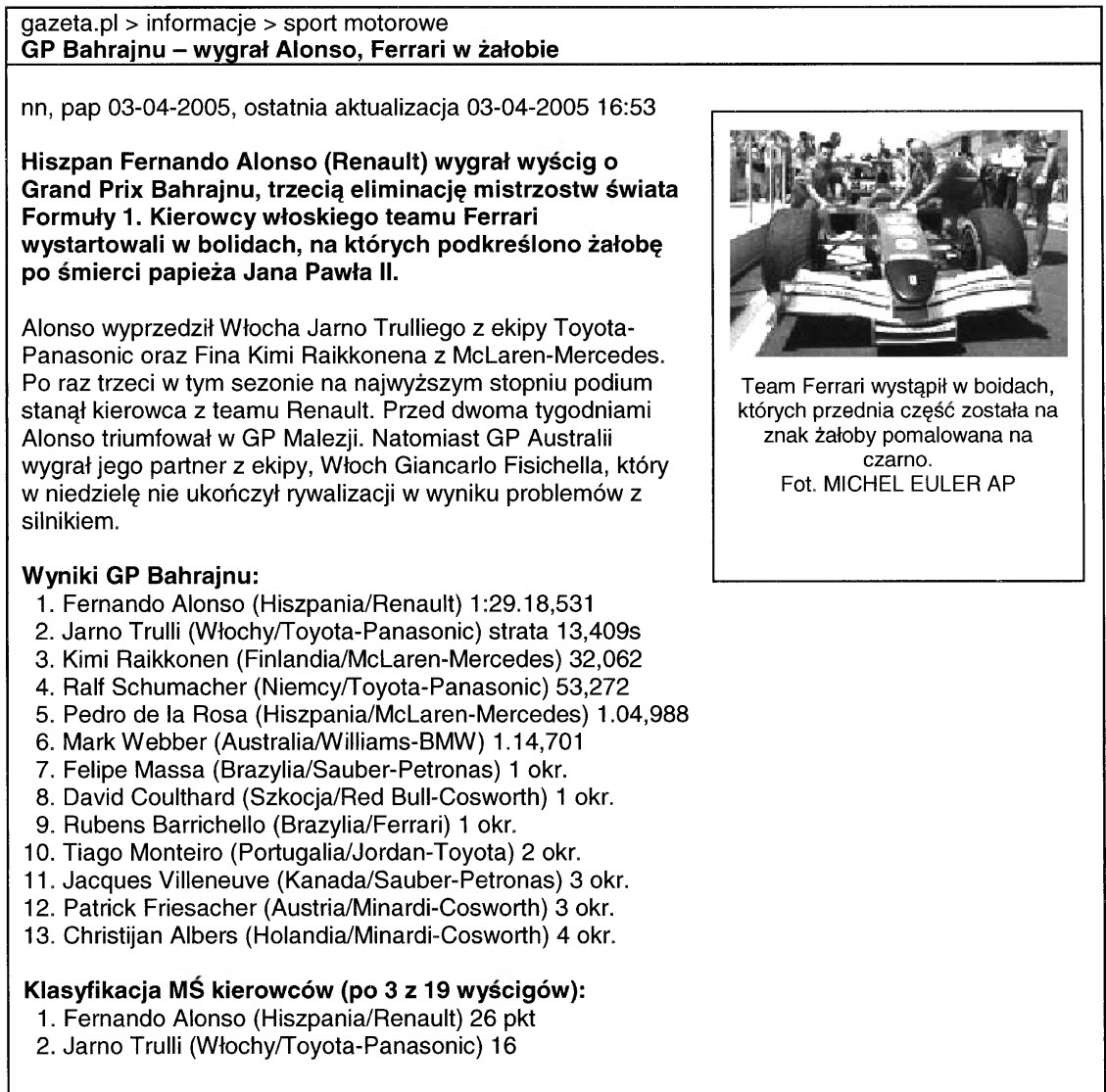

Abb. 2: Polnischer Beispieltext aus Sport Gazeta (http://sport.gazeta.pl/moto/1,35382, 2635324.html-04.04.2005)

Die Sprache ist den brasilianischen Studenten völlig fremd, die Textsorte (Zeitungsartikel) und das Thema (Formel 1) sind ihnen jedoch bekannt. In einem ersten Schritt würde man den Studenten nur die erste Hälfte des Textes ohne die Liste der Platzierungen präsentieren. Bei einer ersten, oberfläch- 
lichen Betrachtung lassen sich Textsorte (Internet-/Zeitungsartikel) und Thema (Formel-1-Rennen) verifizieren. Hierdurch wird den Studierenden intuitiv die Strategie des globalen Lesens vorgestellt.

Im nächsten Schritt werden anhand des Vorwissens über das Textsortenmuster in der Muttersprache gemeinsam Erwartungen darüber gesammelt, welche Informationen der Text beinhalten muss bzw. kann (z. B.: Gewinner/ Team, Ort der Veranstaltung usw.). Sie werden in einer Tabelle geordnet. Hiermit beginnt schon der Übergang zum selektiven Lesen. Die Studenten suchen nun nach weiteren Elementen, die zum Textverständnis beitragen:

- Eigennamen

- Daten und Uhrzeiten

- Internationalismen: Wörter, die in vielen Sprachen ähnlich sind (Information, Club usw.

- Wörter, die sich auf Grund ihrer Ähnlichkeiten mit Wörtern der Muttersprache oder anderer, schon bekannter Fremdsprachen (z. B. Englisch oder Französisch) erschließen lassen.

Mit Portugiesisch als Muttersprache könnten die Ergebnisse etwa folgendermaßen aussehen:

Eigennamen: $\quad$ Fernando Alonso; Renault; Ferrari; Jarno Trulli usw.

Datum, Uhrzeit: $\quad 3-04-2005$ 16:53

Internationalismen: Grand Prix; GP; Formuty 1, podium, sport

Ähnliche Wörter: Portugiesisch:

gazeta (,gazeta“ - kleine Zeitung); aktualizacja ( „atualizar“ - aktualisieren); trzecia („terceira" - dritte); eliminację („eliminatória“" Ausscheidungskampf); hiszpan („,hispânico“-spanisch); ekipy (,equipe“Mannschaft); triumfowat („triunfar" - triumphieren); rywalizacji („rival“ - Rivale); problemów (,problema“ - Problem); informacje (,informação" - Information); Malezji („Malásia“ - Malaysia); Australii („Austrália“ - Australien)

Englisch: $\quad$ teamu (team); motorowe (motor); sezonie (season)

Die Ergebnisse werden gesammelt und im Plenum diskutiert, so dass die Studenten ein Feedback zur Aufstellung und Überprüfung ihrer Hypothesen bekommen. Auf diese Weise würde man etwa zu folgenden Ergebnissen kommen: 


\begin{tabular}{|l|l|}
\hline Was? & Formel 1-GP Bahrain \\
\hline Wo? & Bahrain \\
\hline Wer? & \\
1. Platz & Fernando Alonso - Renault \\
2. Platz & Jarno Trulli - ToyotalPanasonic \\
3. Platz & Kimi Raikonnen - McLaren/Mercedes \\
\hline Weitere Details: & \\
- Zeiten? & $-?$ \\
- Ferrari? (Michael Schumacher/ & \\
Rubens Barrichello) & - Panne? ausgeschieden? \\
- ... & - drittes Podium fïr Renault in der Saison \\
\hline
\end{tabular}

Danach erst würden die Studenten die Liste der Platzierungen bekommen, anhand deren sie ihre Hypothesen selbst überprüfen und die Tabelle mit den fehlenden Informationen ergänzen könnten.

Aus dieser Unterrichtseinheit sollen die Studenten die Erkenntnis mitnehmen, dass top-down-Lesestrategien für das Lesen in jeder Sprache gültig sind. Das globale und das selegierende Lesen werden ihnen als effektive Strategien für die schnelle Bewertung eines Textes und für die Entnahme von Textinformationen vorgestellt, und das Zusammenspiel von top-down- und bottom-upVerfahren wird von den verschiedenen „Phasen“ der Lektüre (1. globales Lesen; 2. Hypothesenbildung; 3. sprachliche Analyse; 4. Überprüfung der Hypothesen; 5. Diskussion und Feedback) verdeutlicht.

Natürlich kann ein solches heuristisches Verfahren nur mit ganz bestimmten, gut ausgewählten Einzeltexten funktionieren. Insgesamt ist es wohl wirksamer als motivationsfördernder Einstieg denn als echtes Lesetraining. Es macht den Studierenden aber eines gleich zu Anfang klar: Leseverstehen in der Fremdsprache, so schwierig es sein mag, ist dennoch kein aussichtsloses Unternehmen.

\section{Literatur}

Börner, Wolfgang/Vogel, Klaus (1996): Zu Fragen der Wechselwirkung von Textrezeption und Textproduktion beim Fremdsprachenerwerb. Eine Einleitung. In: Börner, Wolfgang/Vogel, Klaus (Hg): Texte im Fremdsprachenerwerb. Tübingen: Narr. S. 1-17.

Ehlers, Swantje (2003): Übungen zum Leseverstehen. In: Bausch, Karl-Richard/Christ, Herbert/Krumm, Hans-Jürgen: Handbuch Fremdsprachenunterricht. Tübingen/Basel: Francke. S. 287-291.

Fabricius-Hansen, Cathrine (2002): Texte in der Fremdsprache lesen und verstehen: Überlegungen zu einem vernachlässigten Thema. In: SPRIKreports - Reports of the project Languages in Contrast (Språk i contrast). No. 16, January 2002. URL: http:// www.hf.uio.no/german/sprik.

Groeben, Norbert/Christmann, Ursula (1996): Textverstehen und Textverständlichkeit aus sprach-/denkpsychologischer Sicht. In: Börner, Wolfgang/Vogel, Klaus: Texte im Fremdsprachenerwerb. Tübingen: Narr. S. 67-89. 
Häussermann, Ulrich/Piepho, Hans-Eberhard (1996): Aufgabenhandbuch Deutsch als Fremdsprache: Abriss einer Aufgaben- und Übungstypologie. München: Iudicium.

Rogério, Rosa Helena de Freitas (2003): Uso de estratégias de leitura em textos alemães por alunos dos CELs - SP [Anwendung von Lesestrategien auf deutsche Texte von Schülern der öffentlichen Sprachenzentren CELs - SP]. São Paulo: FLLCH-USP (unveröffentlicht).

Solmecke, Gerd (1993): Texte hören, lesen und verstehen. Berlin/München [u.a.]: Langenscheidt.

Wolff, Dieter (1996): Kognitionspsychologische Grundlagen neuer Ansätze in der Fremdsprachendidaktik. In: Info-DaF 5, Okt/1996, S. 541-560. 